\title{
Differential autogrooming response to the tracheal mite Acarapis woodi by the honey bees Apis cerana and Apis mellifera
}

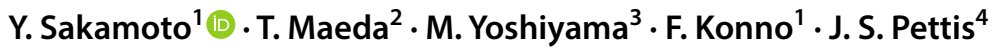

Received: 1 May 2019 / Revised: 18 September 2019 / Accepted: 22 October 2019 / Published online: 22 November 2019

(c) The Author(s) 2019

\begin{abstract}
The infestation of honey bees by the endoparasitic tracheal mite Acarapis woodi was first discovered in Apis mellifera on the Isle of Wight, England, and the mite has since spread to all continents except Australia. Since 2010, this tracheal mite has spread rapidly in the Japanese honey bee, Apis cerana japonica, of mainland Japan, causing considerable colony mortality. In contrast, infestations by the mites in the imported and managed European honey bee, A. mellifera, have rarely been observed in Japan. A previous laboratory experiment revealed a difference in susceptibility by demonstrating that the tracheal mite more frequently enters tracheae of A. cerana than those of A. mellifera. In this study, we compared autogrooming responses of A. cerana and A. mellifera by depositing a mite on each honey bee's mesoscutum, and we then assessed the efficacy of autogrooming to remove the mite. The bees that received mites more frequently showed an autogrooming response compared to unchallenged bees in both bee species. However, a significantly greater proportion of A. mellifera individuals autogroomed compared to A. cerana. In addition, when bees autogroomed, A. mellifera removed the tracheal mite more effectively than $A$. cerana. When considering all bees in the mite-deposited group, the proportion of mite removal in A. mellifera was almost twice as high as that in A. cerana. Thus, the difference in susceptibility to the tracheal mite between these two bee species is attributed to the difference in the behavioral response threshold to mites and the effectiveness of mite removal by grooming.
\end{abstract}

Keywords Emerging infectious disease $\cdot$ Endoparasitic mite $\cdot$ Host-parasite relationship $\cdot$ Japanese honey bee

Significance statement: The tracheal mite is less pathogenic to Apis mellifera than to Apis cerana because A. mellifera more frequently responds to the mite by grooming and more effectively removes it.

Electronic supplementary material The online version of this article (https://doi.org/10.1007/s00040-019-00732-w) contains supplementary material, which is available to authorized users.

Y. Sakamoto

sakamoto.yoshiko@nies.go.jp

1 National Institute for Environmental Studies, 16-2 Onogawa, Tsukuba, Ibaraki 305-8506, Japan

2 Institute of Agrobiological Sciences, National Agriculture and Food Research Organization, 1-2 Ohwashi, Tsukuba, Ibaraki 305-0851, Japan

3 Institute of Livestock and Grassland Science, National Agriculture and Food Research Organization, 2 Ikenodai, Tsukuba, Ibaraki 305-0901, Japan

4 Pettis and Associates, LLC, Salisbury, MD 21801, USA

\begin{abstract}
Abbreviations
EIDs Emerging infectious diseases

LT Local time

AIC Akaike information criterion
\end{abstract}

\section{Introduction}

Emerging infectious diseases (EIDs) are defined as diseases that have recently increased in incidence, geographical range, or host range; have changed pathogenesis; or have been discovered or newly recognized (Lederberg et al. 1992; Morse 1993; Daszak et al. 2000). A majority of EIDs are caused by a change in host-parasite ecology. A so-called host-parasite evolutionary arms race has been found in many biological systems: parasites adapt to increase their success rate of parasitism, whereas hosts adapt to prevent parasitism or reduce pathogenicity (Forfert et al. 2015). Under the current trends of globalization, however, more and more exotic species are being introduced into new locations via numerous transport networks (Banks et al. 2015), which allows parasites or pathogens to encounter novel hosts (Daszak 
et al. 2001). Such introductions have a particularly great impact because the new hosts do not have effective countermeasures to prevent parasitism or reduce pathogenicity.

In honey bees, many EIDs involve interactions between naïve hosts and introduced parasites, because international trade in domesticated bees and bee products has increased over the past several decades (Mutinelli 2011). For instance, the ectoparasitic mites Varroa destructor and Tropilaelaps mercedesae native to Asia and the small hive beetle Aethina tumida native to Africa are harmful to subspecies of the European honey bee, Apis mellifera (Neumann and Ellis 2008; Nazzi and Le Conte 2016; Chantawannakul et al. 2018). These EIDs are some of the factors behind the decline of honey bee populations seen mainly in northwestern Europe and North America, which is in turn causing a decline in pollination service (Fürst et al. 2014; Dicks et al. 2016).

The infestation of honey bees by the endoparasitic tracheal mite Acarapis woodi was first associated with a condition that caused considerable colony mortality in $A$. mellifera on the Isle of Wight, England, in the early 1900s (Rennie 1921), and the mite has since spread to all continents except Australia (Sammataro et al. 2000). The tracheal mite is approximately $125-175 \mu \mathrm{m}$ in length (DelfinadoBaker and Baker 1982) and spends its life in a trachea of a single host (Sammataro and Needham 1996). Both larval and adult mites pierce the honey bee's tracheal walls to feed on the hemolymph (Pettis and Wilson 1996). Mated females leave the trachea and migrate to a trachea of a newly emerged bee to start reproducing (Sammataro and Needham 1996). The mite's population size and prevalence increase in winter, probably because of increased longevity of bees during this season and increased clustering with cooler temperatures (Otis and Scott-Dupree 1992). Colonies heavily infested with $A$. woodi show greater mortality (Bailey and Lee 1959), low honey production (Eischen et al. 1989), and thermoregulatory disorder in cool climates (Eischen 1987; McMullan and Brown 2009), due to damage of the bees' tracheal systems.

Acarapis woodi was first recorded in Japan in 2010 (Ministry of Agriculture, Forestry and Fisheries 2019). In Japan, there are two Apis species, the native Japanese honey bee, Apis cerana japonica, and the European honey bee, A. mellifera, which is a non-native and managed species. The mite has spread rapidly in A. cerana over a wide range of the country (37 of 47 prefectures, totaled from Maeda 2015; Maeda and Sakamoto 2016; Ministry of Agriculture, Forestry and Fisheries 2019) and brought about heavy damage in winter (Maeda and Sakamoto 2016). In contrast, $A$. woodi has been observed in only a few colonies of A. mellifera in Japan (Kojima et al. 2011; Ministry of Agriculture, Forestry and Fisheries 2019; Maeda, unpublished data). That difference of susceptibility between Apis species was demonstrated by laboratory experiments showing that tracheal mites more frequently enter tracheae of $A$. cerana than those of A. mellifera (Sakamoto et al. 2016). Some researchers have proposed that the infestation rate decreases if honey bees sufficiently remove the tracheal mite before it migrates into a new host and that autogrooming is involved in mite removal (Danka and Villa 2003; Sakamoto et al. 2016). Autogrooming with the pro- and/or mesothoracic legs enables bees to remove ectoparasites (Boecking and Spivak 1999; Danka and Villa 2005).

In this study, we conducted an experiment to compare autogrooming responses of $A$. cerana and A. mellifera by depositing a mite on the mesoscutum of adult worker bees and then assessing the efficacy of autogrooming to remove the mite. In addition, we examined which factors are involved in the success of mite removal. Based on our observations, we discuss why only A. cerana suffers from heavy infestation of the tracheal mite in Japan.

\section{Materials and methods}

\section{Honey bee colonies}

We utilized three colonies of A. cerana japonica and three colonies of A. mellifera. The colonies of A. cerana were captured as swarms from the field in the spring of that year. The colonies of A. mellifera were unrelated and were a crossbred race derived mainly from $A$. m. ligustica. First- and secondyear queens were mixed in the A. mellifera colonies. Apis mellifera colonies were kept in Langstroth hives, whereas $A$. cerana were kept in smaller movable-frame $(205 \times 215 \mathrm{~mm})$ hives similar to Langstroth hives to allow for the removal of frames containing emerging bees for this study. All colonies were kept at our institutes in Tsukuba City, Ibaraki Prefecture, Japan. To eliminate the effects of acaricides on tracheal mites, no treatments for varroa mites were administered for at least 6 months before starting the experiments in all colonies used in this study, although such treatments are ordinarily administered in colonies of A. mellifera in Japan.

\section{Preparation for the experiment}

The experiment was prepared and conducted at our laboratory in October and November 2015. Brood combs were cut into small pieces, and each was placed in a 480-ml clear plastic cup and kept in a dark incubator to allow for adult bee emergence $\left(35^{\circ} \mathrm{C}, 55 \% \mathrm{RH}\right)$. Emerged bees $(0-24 \mathrm{~h}$ old) were removed from combs at 14:00 local time (LT). They were placed individually in a $120-\mathrm{ml}$ clear plastic cup (64 $\mathrm{mm}$ in diameter at the bottom) with a filter paper on the bottom. The bees were provided with cotton wool kept saturated with $50 \%$ sucrose solution $(\mathrm{v} / \mathrm{v})$ via a $1.5-\mathrm{ml}$ 
microcentrifuge tube. $\mathrm{CO}_{2}$ narcosis was not used because it induces autogrooming behaviors in honey bees when they recover from narcosis (personal observation). When we found varroa mites on a bee, we removed them with forceps because they induce grooming behaviors. The separated bees were kept in another dark incubator $\left(25^{\circ} \mathrm{C}, 55 \% \mathrm{RH}\right)$ until the start of the experiment the next day. A total of 713 bees (71, 142, and 131 from each colony of A. cerana, 186, 35, and 148 from each colony of $A$. mellifera) were observed throughout the experiment.

\section{Depositing mites on mesoscutum of adult honey bees and behavioral observations}

Tracheal mites used for the experiment were provided from three highly infested $A$. cerana colonies kept by a bee keeper or caught from the field in Tsukuba City. Foraging bees were caught at the entrance of a hive and kept in a cage at $25^{\circ} \mathrm{C}$ in our laboratory. Infested tracheae used on the day of an experiment were removed from bees and deposited on a glass slide on moist filter paper in a Petri dish, so as not to dry out the tracheae. Just before starting an observation, a mite was removed from a trachea on the tip of a human eyelash mounted on a stick. Only female mites were used for the experiment.

We conducted the experiment between 08:00 and 18:00 LT. At this time, the average bee's age was approximately $35 \mathrm{~h}$ (range 18-52 h). Based on observations that young bees aggregate and seem to show thermotaxis, the plastic cup with a focal bee was placed on a slide warmer held at $35^{\circ} \mathrm{C}$ in the $25^{\circ} \mathrm{C}$ laboratory. This method encourages bees to stay at the bottom of the cup (floor-heating method, Figs. S1, S2). Bees that were not stationary were not used for the experiment because it was too difficult to deposit mites on the bees.

A mite was deposited on either the left or right side of the bee's mesoscutum with the eyelash brush. Whether to deposit a mite on the left or right side was randomly determined by throwing a die each time. Control bees were simply stimulated by stroking once on the mesoscutum with the brush. Separate brushes were used for the mite-deposited and control groups. After depositing a tracheal mite, we observed the bee's responses for $7 \mathrm{~min}$, within which autogrooming responses to mites were sufficiently observed and mites did not enter spiracles, based on preliminary observations (Danka and Villa 2003). Moving a mesothoracic leg anteriorly across the mesoscutum was regarded as grooming behavior in this experiment (hereafter "GR"; Movie 1). We recorded the number of GR attempts, time to initial GR, and the positions (i.e., treated or untreated side). The performance of a grooming invitation dance by a worker rapidly shaking her abdomen, which attracts the attention of nestmates (Ruttner and Hänel 1992), was also recorded. After the observation period, we held the bee's body and wings by inserting forceps between the thorax and abdomen to prevent the mite from falling as the bee flapped its wings and confirmed whether the mite was removed or not under a microscope. Bees were not re-used in another assay.

\section{Statistical analyses}

The proportions of bees that showed GR (hereafter "GR bee") and the proportions of mite removal were compared using the Tukey-type multiple comparison test for proportions (Zar 1999). The number of GR attempts was compared between the treated and untreated sides by a Wilcoxon rank sum test to investigate whether bees respond to the position of a mite. Factors associated with successful mite removal were estimated with generalized linear models (McCullagh and Nelder 1989) in GR individuals. These models assumed a binomial distribution and used a logistic link function. The response variable was presence of the mite on the body at the end of an experiment, and the explanatory variables were the time to initial GR (s) on the treated side, the number of GR attempts on the treated side, bee species (dummy variable), and their interactions. The model with the lowest Akaike's information criterion (AIC) was chosen as the final model.

\section{Results}

\section{GR response to the tracheal mite}

In each control group of A. cerana and A. mellifera, the proportion of GR bees was approximately $20 \%$ (Fig. $1, p>0.05$, Tukey-type multiple comparison test for proportions). The

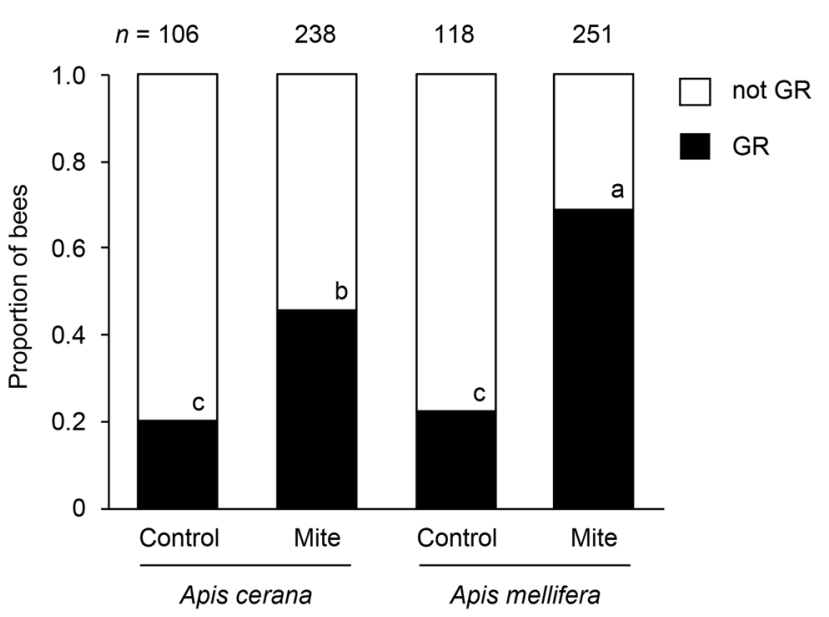

Fig. 1 Proportions of honey bees grooming their mesoscutum with their mesothoracic leg (GR) in control and mite-deposited groups of Apis cerana and Apis mellifera. Proportions followed by the same letter are not significantly different by the Tukey-type multiple comparison test $(p>0.05)$ 
proportion of GR bees in the mite-deposited group was significantly greater than those of the control group in both bee species, and the proportion of GR bees in A. mellifera (69\%) was greater than that in A. cerana (45\%) (Fig. $1 ; p<0.05$, Tukey-type multiple comparison test for proportions). In the mite-deposited group, the grooming invitation dance was observed only in A. cerana (5.7\%). In the control group, the dance was not observed in either bee species.

\section{Recognition of the mite's position}

In each control group of $A$. cerana and A. mellifera, there was no difference in the number of GR attempts between the treated and untreated sides ( $p>0.05$, Wilcoxon rank sum test; Fig. 2). In the mite-deposited group, the number of GR attempts on the treated side was significantly higher than that on the untreated side in both A. cerana and A. mellifera $(p<0.001$, Wilcoxon rank sum test).

\section{Effectiveness of GR to remove the tracheal mite}

Compared to those not showing GR, when bees in the mitedeposited group showed GR on the treated side, the proportions of mite removal increased in both bee species (Fig. 3). However, the proportion of mite removal in A. mellifera (77\%) was significantly higher than that in A. cerana $(57 \%)$ in GR individuals $(p<0.05$, Tukey-type multiple comparison test for proportions). When considering all bees in the mite-deposited group (i.e., both GR and not GR), the proportion of mite removal in A. mellifera (59\%) was almost twice as high as that in A. cerana $(33 \%)(p<0.001$, Chi-squared test, $\chi^{2}=32.67, d f=1$ ).

\section{Factors associated with the removal of tracheal mites}

According to the generalized linear model with the lowest AIC value, the explanatory variables time to initial GR, bee species, and no interactions were selected (Fig. S3); short time to initial GR and A. mellifera affected the success of mite removal (Table 1). The models with the 10 lowest AIC values were close, and all of them included bee species (Fig. S3).

\section{Discussion}

\section{Difference in response behaviors between $A$. cerana and $A$. mellifera}

A tracheal mite placed on the mesoscutum induced GR in both A. cerana and A. mellifera, indicating that bees of both species were able to recognize the presence of the mite. However, the proportion of GR bees in A. mellifera was significantly greater than that in A. cerana (Fig. 3), suggesting that the behavioral response threshold of A. mellifera to the
Fig. 2 Number of grooming attempts of honey bees during the experiment on treated and untreated sides in control and mite-deposited groups of Apis cerana and Apis mellifera. Box plots present the lower quartile, median, and upper quartile, and whiskers represent 1.5 times the interquartile range. Differences were compared by Wilcoxon rank sum test

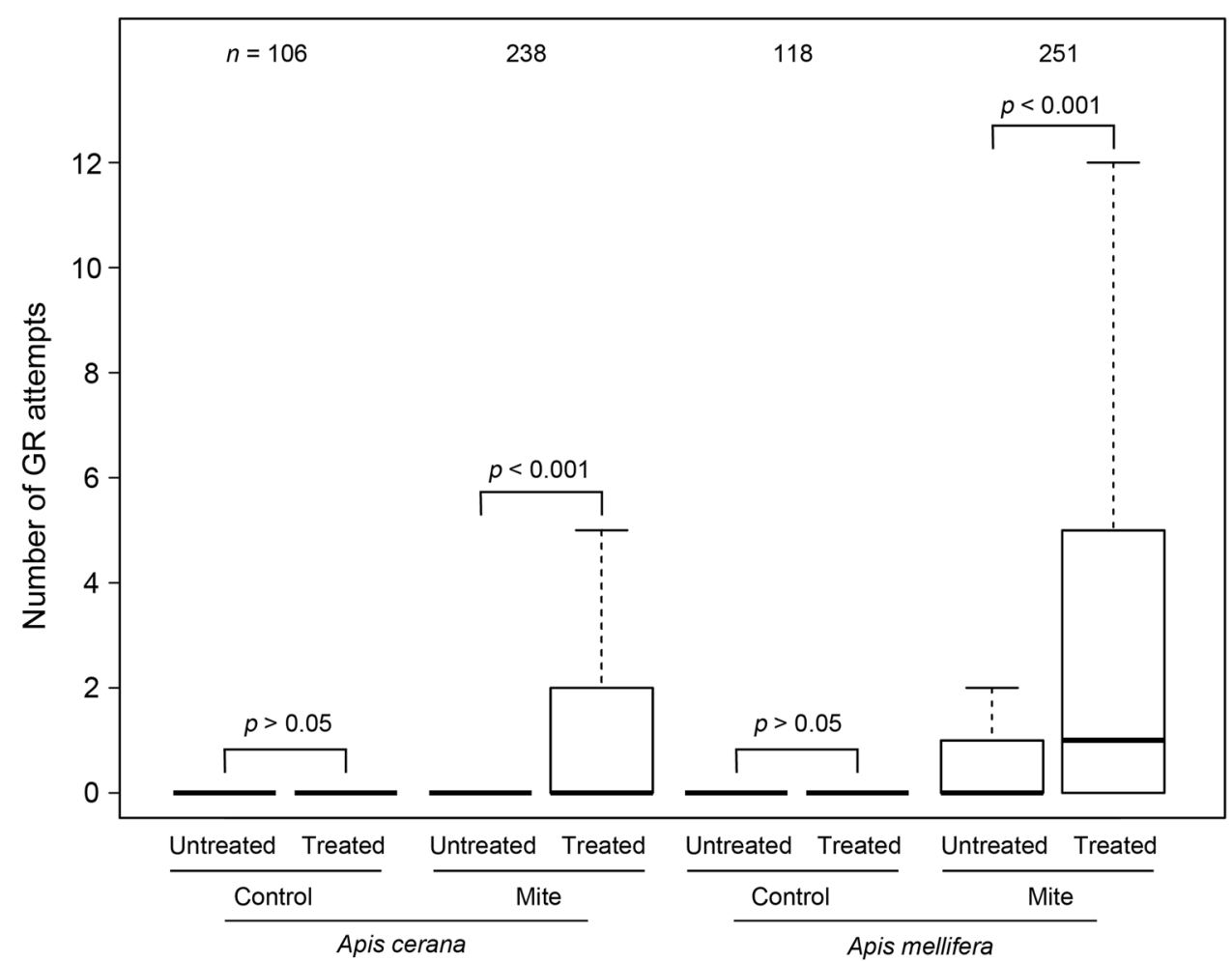



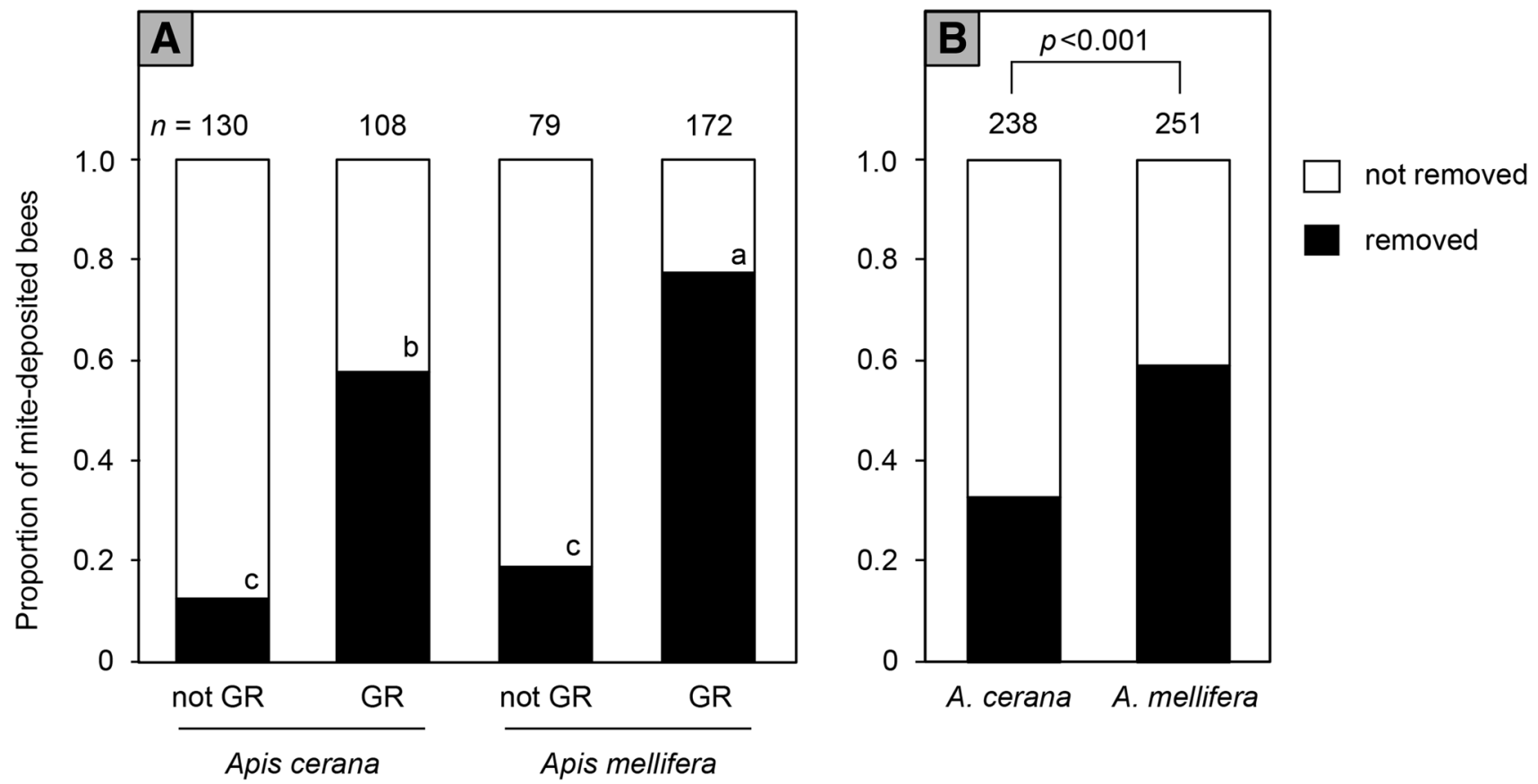

Fig. 3 In the mite-deposited group, proportions of honey bees on whose mesoscutum a tracheal mite was removed in Apis cerana and Apis mellifera (a) when bees groomed (GR) or did not groom (not $\mathrm{GR}$ ) and $\mathbf{b}$ in total. In $\mathbf{a}$, proportions followed by the same letter are

not significantly different by the Tukey-type multiple comparison test $(p>0.05)$. In $\mathbf{b}$, the Chi-squared test demonstrated a significant difference $(p<0.001)$

Table 1 Result for the best generalized linear model examining the effects of bee species and time to initial GR on the presence of a tracheal mite after the end of experiment

\begin{tabular}{lllll}
\hline Variable & Estimate & SE & $z$ value & $p$ \\
\hline Intercept & -0.798 & & & \\
Bee species, A. mellifera & -0.842 & 0.282 & -2.982 & $0.003^{* *}$ \\
Time to initial GR (s) & 0.003 & 0.001 & 2.473 & $0.013^{*}$ \\
\hline
\end{tabular}

${ }^{*} p<0.05, * * p<0.01$

mite was lower than that of A. cerana. When exposed to a tracheal mite, both bee species groomed intensively on the side of their mesoscutum on which the mite was deposited. One explanation of this behavior is that the bees responded to the physical stimulus of the tracheal mite. A chemical stimulus is also a possible explanation, however, as both bee species have been shown to detect varroa mites by chemical signals and remove them from brood cells (Martin et al. 2002).

\section{Difference in proportions of mites removed between $A$. cerana and $A$. mellifera}

In both species, tracheal mites were absent from approximately 60 to $80 \%$ after the 7 -min assay when bees showed GR, indicating that autogrooming on the mesoscutum was

an effective way to remove tracheal mites (Fig. 3). This finding was also reported by Danka and Villa (1998), who showed that the midleg of $A$. mellifera plays an important role in removing tracheal mites. The lower stimulus threshold for behavioral responses of A. mellifera may facilitate its ability to detect and remove the tracheal mite rapidly from the body. Our results also demonstrated that GR individuals of A. mellifera removed tracheal mites significantly more effectively than those of A. cerana.

The best model indicated that the time to initial GR and bee species are significant factors in the effectiveness of GR. With regard to time to initial GR, the earlier that a bee detects and grooms tracheal mites, the higher the proportion of mites removed. With a time delay, it is likely to become difficult to remove a mite if it hides deep within the bee's hairs or in the groove at the edge of the mesoscutum. However, the effect of early detection did not differ between bee species. The number of GR attempts was not selected as a factor in the best model. The result could be attributed to two opposite aspects of this factor: mite removal is facilitated by increasing the number of GR attempts, whereas the number of GR attempts increases if the mite is not removed. Bee species definitely affected the removal efficacy because the models with the 10 lowest AIC values all included bee species. Apis mellifera can remove tracheal mites more effectively than A. cerana by some behavioral differences that 
were not reflected in time and number of GRs and/or some morphological differences between the bee species.

\section{What is happening in the natural environment?}

In the present experiment, there was no difference in GR response between A. cerana and A. mellifera without mites. Unlike the experimental conditions with a single worker bee, there are nestmates in the natural environment of the hive. Danka and Villa (2003) reported that 46-52\% of A. mellifera individuals with their nestmates autogroomed on their mesoscutum compared to $26 \%$ without nestmates in the present experiment when a tracheal mite was not deposited. This finding indicates that the presence of nestmates may also trigger bees to autogroom. Moreover, in our previous colony-level assay (Sakamoto et al. 2016), the frequency of GR was higher in A. mellifera than A. cerana without tracheal mites. Although the presence of nestmates may increase the frequency of autogrooming, the threshold of A. mellifera was lower than that of A. cerana, so we infer that the frequency of autogrooming would be higher in A. mellifera than in A. cerana in the natural environment.

Honey bees also can be helped by their nestmates to remove mites from their bodies. Such allogrooming normally involves a dancing bee and a bee that responds to the dance by grooming the dancer (Milum 1955). Pettis and Pankiw (1998) showed that frequencies of the grooming invitation dance and allogrooming were higher in colonies with larger tracheal mite infestations. In the present study, the grooming invitation dance was observed in only A. cerana. It is unclear whether allogrooming is effective at removing tracheal mites, but this conspecific behavioral difference may be another factor related to tracheal mite infestation. Further work is needed to explore this question in more detail.

\section{Why does the behavioral difference occur?}

To discuss the behavioral differences seen in the current study, we refer to the relationship between $V$. destructor and honey bees. The varroa mite was originally an ectoparasitic mite of the Asian honey bee, A. cerana. However, A. mellifera colonies became infested by the varroa mite from $A$. cerana in the 1950 s, and subsequently the mite has spread rapidly to A. mellifera by transporting productive colonies. The varroa mite does not cause significant problems in the original host A. cerana, but it can infest the new host $A$. mellifera at higher density and has become a global pandemic. This susceptibility difference is partly related to the behavioral difference between the two bee species. Apis cerana damages varroa mites with autogrooming and allogrooming and in so doing frequently removes them (Peng et al. 1987;
Büchler et al. 1992). By contrast, similar behavior has rarely been seen in A. mellifera (Pritchard 2016).

Why were damages by the tracheal mite not observed in A. mellifera in Japan? Based on our results, we conclude that the pathogenicity of the tracheal mite to A. mellifera is lower than that to $A$. cerana because A. mellifera more frequently responds to the mite than does $A$. cerana and more effectively removes it. In fact, little infestation of the tracheal mite in A. mellifera outside the laboratory has been reported in Japan except for a few colonies (Maeda and Sakamoto 2016, unpublished data). We speculate that resistant colonies of A. mellifera have been distributed across a wide area of Japan since at least 2010. The tracheal mite has spread worldwide and brought severe problems to A. mellifera in the new locales, but significant declines in the incidence of the tracheal mite in the decades since introduction have been observed in Britain (Morison et al. 1965), the United States (Moore et al. 2015), and elsewhere. The declines likely reflect the selection of some genetically resistant strains (Page and Gary 1990; Lin et al. 1996; Danka 2001; van Engelsdorp and Otis 2001; Danka and Villa 2003; Bourgeois et al. 2015)

Japan has imported A. mellifera colonies from other countries including Australia (Fig. S4). A large number of resistant strains are considered to have been imported into Japan through those regions, although the main routes are unknown. This may explain why there are few observations of the mite infestation in A. mellifera in Japan. In contrast, $A$. cerana japonica does not have sufficiently effective grooming behavior at this time. Although the origin of tracheal mites found in Japan is debatable, some genetic analyses (Kojima et al. 2011; Sakamoto et al. unpublished data) and the year of discovery imply that the tracheal mites were introduced from overseas. Colonies of A. mellifera with low infestation rates of the tracheal mites could have passed through quarantine. Artificial movement of honey bee colonies has changed the host-parasite relationship and caused the EID in A. cerana. In the future, A. cerana may be able to overcome the damage of the tracheal mite as has been seen in A. mellifera. However, the acquisition of resistance may be affected by, for example, some species-specific morphological differences associated with mite removal and the presence or absence of artificial effects such as managed or wild bees. We plan to further analyze factors related to susceptibility to the tracheal mite that will help predict the future consequence of tracheal mite introduction to $A$. cerana.

Acknowledgements We are grateful to Shigeki Kishi of the Institute of Agrobiological Sciences, National Agriculture and Food Research Organization, for his assistance in performing statistical analyses; to Mio Nishiyama of the National Institute for Environmental Studies and Yoshio Suzuki, Chizuko Yoshida, Akira Kawada, and Jun Arai of the Kawakami Farm Co., Ltd. for their assistance in preparing experiments; 
to Yudai Okuyama of Tsukuba Botanical Garden, National Museum of Nature and Science, and Kunihiko Numajiri in Tsukuba City for helping to provide bee samples; to Benjamin P. Oldroyd of the University of Sydney and Kiyoshi Kimura of the Institute of Livestock and Grassland Science, National Agriculture and Food Research Organization (NILGS) for giving import and export status information of Australia and Japan, respectively; and to Noriko Takaya of NILGS for her assistance in aggregating information about the importation of honey bee colonies. We also thank Koichi Goka, Makihiko Ikegami, Hironori Sakamoto, Makiko Okudaira, and our colleagues at National Institute for Environmental Studies for their helpful cooperation. This study was supported by Grants-in-Aid for Scientific Research 26290074 from the Japan Society for the Promotion of Science.

Author contributions YS designed the experiments; YS, TM, MY, and FK collected and maintained experimental samples; FK and YS collected experimental data; YS participated in statistical analysis; all authors helped draft the manuscript.

\section{Compliance with ethical standards}

Conflict of interest Authors declare no competing interest.

Open Access This article is distributed under the terms of the Creative Commons Attribution 4.0 International License (http://creativeco mmons.org/licenses/by/4.0/), which permits unrestricted use, distribution, and reproduction in any medium, provided you give appropriate credit to the original author(s) and the source, provide a link to the Creative Commons license, and indicate if changes were made.

\section{References}

Bailey L, Lee DC (1959) The effect of infestation with Acarapis woodi (Rennie) on the mortality of honey bees. J Insect Parhol 1:15-24

Banks NC, Paini DR, Bayliss KL, Hodda M (2015) The role of global trade and transport network topology in the human-mediated dispersal of alien species. Ecol Lett 18:188-199

Boecking O, Spivak M (1999) Behavioral defenses of honey bees against Varroa jacobsoni Oud. Apidologie 30:141-158

Bourgeois AL, Villa JD, Holloway B, Danka RG, Rinderer TE (2015) Molecular genetic analysis of tracheal mite resistance in honey bees. J Apic Rec 54:314-320

Büchler R, Drescher W, Tornier I (1992) Grooming behavior of Apis cerana, Apis mellifera and Apis dorsata and its effect on the parasitic mites Varroa jacobsoni and Tropilaelaps clareae. Exp Appl Acarol 16:313-319

Chantawannakul P, Ramsey S, vanEngelsdorp D, Khongphinitbunjong K, Phokasem P (2018) Tropilaelaps mite: an emerging threat to European honey bee. Curr Opin Insect Sci 26:69-75

Danka RG (2001) Resistance of honey bees to tracheal mites. In: Webster TC, Delaplane KS (eds) Mites of the honey bee. Dadant and Sons, Hamilton, pp 117-129

Danka RG, Villa JD (1998) Evidence of autogrooming as a mechanism of honey bee resistance. J Apic Res 37:39-46

Danka RG, Villa JD (2003) Autogrooming by resistant honey bees challenged with individual tracheal mites. Apidologie 34:591-596

Danka RG, Villa JD (2005) An association in honey bees between autogrooming and the presence of migrating tracheal mites. Apidologie 36:331-333
Daszak P, Cunningham AA, Hyatt AD (2000) Emerging infectious diseases of wildlife: threats to biodiversity and human health. Science 287:443-449

Daszak P, Cunningham AA, Hyatt AD (2001) Anthropogenic environmental change and the emergence of infectious diseases in wildlife. Acta Trop 78:103-116

Delfinado-Baker M, Baker EW (1982) Notes on honey bee mites of the genus Acarapis Hirst (Acari: Tarsonemidae). Int J Acarol 8:211-226

Dicks LV, Viana B, Bommarco R, Brosi B, Arizmendi MD, Cunningham SA, Galetto L, Hill R, Lopes AV, Pires C, Taki H, Potts SG (2016) Ten policies for pollinators. Science 354:975-976

Eischen FA (1987) Overwintering performance of honey bee colonies heavily infested with Acarapis woodi (Rennie). Apidologie 18:293-304

Eischen FA, Cardoso-Tamez D, Wilson WT, Dietz A (1989) Honey production of honey bee colonies infested with Acarapis woodi (Rennie). Apidologie 20:1-8

Forfert N, Natsopoulou ME, Frey E, Rosenkranz P, Paxton RJ, Moritz RF (2015) Parasites and pathogens of the honeybee (Apis mellifera) and their influence on inter-Colonial Transmission. PLoS One 10:e140337

Fürst MA, McMahon DP, Osborne JL, Paxton RJ, Brown MJF (2014) Disease associations between honeybees and bumblebees as a threat to wild pollinators. Nature 506:7488

Kojima Y, Yoshiyama M, Kimura K, Kadowaki T (2011) PCR-based detection of a tracheal mite of the honey bee Acarapis woodi. J Invertebr Pathol 108:135-137

Lederberg J, Shope RE, Oakes SC (1992) Emerging infections: microbial threats to health in the United States. National Academy Press, Washington, DC

Lin H, Otis GW, Scott-Dupree C (1996) Comparative resistance in Buckfast and Canadian stocks of honey bees (Apis mellifera L.) to infestation by honey bee tracheal mites (Acarapis woodi (Rennie)). Exp Appl Acarol 20:87-101

Maeda T (2015) Infestation of honey bees by tracheal mites, Acarapis woodi, in Japan. J Acarol Soc Jpn 24:9-17 (in Japanese)

Maeda T, Sakamoto Y (2016) Tracheal mites, Acarapis woodi, greatly increase overwinter mortality in colonies of the Japanese honeybee, Apis cerana japonica. Apidologie 47:762-770

Martin C, Provost E, Bagnères A-G, Roux M, Clément J-L, Conte YL (2002) Potential mechanism for detection by Apis mellifera of the parasitic mite Varroa destructor inside sealed brood cells. Physiol Entomol 27:175-188

McCullagh P, Nelder JA (1989) Generalized linear models, 2nd edn. Taylor \& Francis, Abingdon

McMullan JB, Brown MJF (2009) A qualitative model of mortality in honey bee (Apis mellifera) colonies infested with tracheal mites (Acarapis woodi). Exp Appl Acarol 47:225-234

Milum VG (1955) Honey bee communication. Am Bee J 95:97-104

Ministry of Agriculture, Forestry and Fisheries (2019) The outbreak situation of the monitoring epidemic. http://www.maff.go.jp/j/ syouan/douei/kansi_densen/kansi_densen.html. Accessed 17 July 2019 (in Japanese)

Moore PA, Wilson ME, Skinner JA (2015) Honey bee tracheal mites: gone? But not for good. https://articles.extension.org/ pages/74070/honey-bee-tracheal-mites:-gone-but-not-for-good. Accessed 17 July 2019

Morison G, Jeffree E, Murray L, Allen M (1965) Acarine and Nosema diseases of honeybees in Britain, 1925-47. Bull Entomol Res 46:753-759

Morse SS (1993) Examining the origins of emerging viruses. In: Morse SS (ed) Emerging viruses. Oxford University Press, New York, pp 10-28

Mutinelli F (2011) The spread of pathogens through trade in honey bees and their products (including queen bees and semen): 
overview and recent developments. Revue Scientifique et Technique de l'OIE 30:257-271

Nazzi F, Le Conte Y (2016) Ecology of Varroa destructor, the major ectoparasite of the Western honey bee, Apis mellifera. Annu Rev Entomol 61:417-432

Neumann P, Ellis J (2008) Guest Editorial: the small hive beetle (Aethina tumida Murray, Coleoptera: Nitidulidae) distribution, biology and control of an invasive species. J Apic Res 47:181-183

Otis GW, Scott-Dupree C (1992) Effects of Acarapis woodi on overwintered colonies of honey bees (Hymenoptera, Apidae) in New York. J Econ Entomol 85:40-46

Page RE, Gary NE (1990) Genotypic variation in susceptibility of honey bees (Apis mellifera) to infestation by tracheal mites (Acarapis woodi). Exp Appl Acarol 8:275-283

Peng YS, Fang YZ, Xu SY, Ge LS (1987) The resistance mechanism of the Asian honey bee, Apis cerana Fabr, to an ectoparasitic mite, Varroa jacobsoni Oudemans. J Invertebr Pathol 49:54-60

Pettis JS, Pankiw T (1998) Grooming behavior by Apis mellifera L. in the presence of Acarapis woodi (Rennie) (Acari: Tarsonemidae). Apidologie 29:241-253

Pettis JS, Wilson WT (1996) Life history of the honey bee tracheal mite (Acari: Tarsonemidae). Ann Entomol Soc Am 89:368-374
Pritchard DJ (2016) Grooming by honey bees as a component of varroa resistant behavior. J Apic Res 55:38-48

Rennie J (1921) Isle of Wight disease in hive bees. Acarine disease: the organism associated with the disease, Tarsonemus woodi, $\mathrm{n}$. sp. Earth Environ Sci Trans R Soc Edinb 52:768-779

Ruttner F, Hänel H (1992) Active defense against Varroa mites in a Carniolan strain of honeybee (Apis mellifera carnica Pollmann). Apidologie 23:173-187

Sakamoto Y, Maeda T, Yoshiyama M, Pettis JS (2016) Differential susceptibility to the tracheal mite Acarapis woodi between Apis cerana and Apis mellifera. Apidologie 48:150-158

Sammataro D, Needham G (1996) Host-seeking behaviour of tracheal mites (Acari: Tarsonemidae) on honey bees (Hymenoptera: Apidae). Exp Appl Acarol 20:121-136

Sammataro D, Gerson U, Needham G (2000) Parasitic mites of honey bees: life history, implications, and impact. Annu Rev Entomol 45:519-548

van Engelsdorp D, Otis GW (2001) Field evaluation of nine families of honey bees for resistance to tracheal mites. Can Enomol 133:793-803

Zar JH (1999) Biostatistical analysis, 5th edn. Prentice Hall, Englewood Cliffs 